\title{
SYNCHRONIZATION AND STABILITY OF AN ELASTICALLY COUPLED TRI-ROTOR VIBRATION SYSTEM
}

\author{
Yonguun Hou, Minguen Du, Pan Fang, Liping Zhang \\ School of Mechanical Engineering, Southwest Petroleum University, Chengdu, China \\ e-mail: ckfangpan@126.com (Pan Fang - corresponding author)
}

\begin{abstract}
A new mechanism, an elastically coupled tri-rotor system, is proposed to implement synchronization. It is composed of a rigid body, three induction motors, coupling unit and springs. According to the Lagrange equation, the model of the system is established. The average method of small parameters is applied to study the synchronization characteristics of the system, therefore, the balance equation and stability criterion of the system can be obtained. Obviously, many parameters affect the synchronous state of the rotors, especially the spring stiffness, the stiffness of the coupling unit and the installation location of the system. Finally, computer simulations are used to verify the correctness of theoretical analysis.
\end{abstract}

Keywords: tri-rotor, synchronization characteristics, stability, computer simulations

\section{Introduction}

The synchronization phenomenon is common in nature. General definitions of synchronization were presented by Blekhman et al. $(1997,2002)$. The synchronization phenomenon is considered as an adjustment of rhythms of oscillating objects due to their internal weak couplings. Dutch scholar Huygens was first to discover the synchronization phenomenon, the synchronous motion of a pendulum hanging on the common base in 1665 (Huygens, 1673). In 1960s, Blekhman proposed the synchronization theory of vibrating machines with two or multiple exciters and successfully solved many engineering problems related to self-synchronization (Blekhman, 1998; Blekhman et al., 1997). Many fields, such as the modeling of nonlinear dynamics, coupling pendulums, mechanical rotors, have attracted attention of reserchers. In dynamics of coupled pendulums and rotors, Blekhman proposed the Poincaré method for the synchronization state and stability. Now it is a method widely used in engineering (Jovanovic and Koskhin, 2012). Based on Blekhman's method, many scientists have developed other methods to analyze synchronization of rotors (Blekhman, 1988). Koluda et al. (2014a,b) derived synchronization conditions and explained observed types of synchronization for coupled double pendula. They used an energy balance method to show how the energy is transferred between the pendula via an oscillating beam. For synchronization of mechanical rotors, Zhao et al. (2010) and Zhang et al. (2012) proposed an average method of modified small parameters, which was applied to study of synchronous multiple unbalanced rotors (Zhang et al., 2013). Hou (2007) studied the synchronism theory of three motors using the Hamilton principle. Balthazar (2004) and Balthazar et al. (2005) described self-synchronization of two and four non-ideal rotating unbalanced motors via numerical simulations. For synchronization and modeling of nonlinear dynamics, a mechanism of interaction between two non-linear dissipative oscillators was presented by Rui (2014). Two pendulums coupled with a weak spring were proposed by Blekhman (1988). Kumon et al. (2002) showed the synchronization phenomenon by designing the controller with applying speed the Gradient Energy method. Fradkov and Andrievsky (2007) focused on the study of phase relations between coupled oscillators. 
However, for synchronization of three non-identical coupled exciters, the phase difference of co-rotating motors stabilizes around $120^{\circ}$ (Zhang et al., 2013). This results in a weakened amplitude of the center of mass. In order to improve vibration amplitude and screening efficiency of the system, three rotors coupled with a weak spring are proposed in this paper. To explore coupling characteristics of the system, synchronization conditions and the synchronous stability criterion of the system are derived with the Poincaré method. Finally, computer simulations are implemented to verify the results of theoretical analysis. It is demonstrated that the spring stiffness, the coupling spring and the installation location plays a significant role in the vibration system.

This paper is organized as follows. The analysis strategy and considered model are described in Section 2. In Section 3, the synchronization condition and the synchronization stability criterion are obtained. In Section 4, the results of numerical simulations and results of the computer simulations are presented, which validate correctness of the theoretical model of the vibration system. Finally, the results are summarized in Section 5.

\section{Model description}

\subsection{Strategy}

The equations of motion for the considered rotation system are as follows (Fang et al., 2015)

$$
\begin{aligned}
& J_{s} \varphi_{s}=\mu \Phi_{s}\left(\varphi_{s}, \ddot{x}\right) \quad s=1, \ldots, k \\
& \ddot{x}+2 \omega_{x} \xi_{x} \dot{x}+\omega_{x}^{2} x=\sum_{j=1}^{k} F_{j}\left(\omega t, \alpha_{1}, \ldots, \alpha_{k}\right)+\mu F_{k+1}\left(\omega t, \alpha_{1}, \ldots, \alpha_{k}\right)
\end{aligned}
$$

where $\mu \Phi_{s}=T_{m s}-T_{f s}, \mu$ is the small parameter, $J_{s}$ is the rotational inertia of $s$-th induction motor, $T_{m s}$ is the driving torque of the induction motors, $T_{f s}$ is the mechanical damping torque of the induction motors, $\xi_{x}$ and $\omega_{x}$ are the damping coefficient and the natural frequency of the system in the $x$-direction, $\omega$ and $\varphi_{s}$ are mechanical velocity and rotation angles of the $s$-th unbalanced rotor.

In the synchronous state, the velocity of the rotors is assumed as $\omega$. Steady forced vibrations with $T=2 \pi / \omega$ are determined by

$$
x=x\left(\omega t, \alpha_{1}, \ldots, \alpha_{k}\right)
$$

Considering that the rotors are uniformly rotating with an initial phase $\alpha_{1}, \ldots, \alpha_{k}$, then the phase angle of rotors should satisfy the synchronous solutions from the second formula Eq. (2.2)

$$
\varphi_{s}=\varphi_{s}^{0}=\omega t+\alpha_{s}
$$

The above-mentioned basic equation may be satisfied with such values of constants $\alpha_{1}, \ldots, \alpha_{k}$

$$
P_{s}\left(\alpha_{1}, \ldots, \alpha_{k}\right)=\mu\left\langle\Phi_{s}\left(\varphi_{s}\left(\varphi_{s}, \ddot{x}\right)\right)\right\rangle=0
$$

Here, the angle brackets $\langle *\rangle$ show the average value for one period by the variable $t$, and the symbol $*$ represents a function related to time $t$

$$
\langle *\rangle=\frac{1}{T} \int_{0}^{T} * d t
$$


If a certain set of constants $\alpha_{1}, \ldots, \alpha_{k}$ is satisfied by Eq. (2.4), all the roots $\chi$ of the algebraic equation

$$
\left|\begin{array}{cccc}
\frac{\partial\left(P_{1}-P_{k}\right)}{\partial \alpha_{1}}-\chi & \frac{\partial\left(P_{1}-P_{k}\right)}{\partial \alpha_{2}} & \cdots & \frac{\partial\left(P_{1}-P_{k}\right)}{\partial \alpha_{k-1}} \\
\frac{\partial\left(P_{2}-P_{k}\right)}{\partial \alpha_{1}} & \frac{\partial\left(P_{2}-P_{k}\right)}{\partial \alpha_{2}}-\chi & \ldots & \frac{\partial\left(P_{2}-P_{k}\right)}{\partial \alpha_{k-1}} \\
\cdots & \cdots & \ldots & \cdots \\
\frac{\partial\left(P_{k-1}-P_{k}\right)}{\partial \alpha_{1}} & \frac{\partial\left(P_{k-1}-P_{k}\right)}{\partial \alpha_{2}} & \ldots & \frac{\partial\left(P_{k-1}-P_{k}\right)}{\partial \alpha_{k-1}}-\chi
\end{array}\right|=0
$$

would have negative real parts, then unique constant values $\alpha_{1}, \alpha_{2}, \ldots, \alpha_{k}$ are determined when the parameter $\mu$ is sufficiently small. Meanwhile, there exists an asymptotic periodic solution to Eq. (2.1). Only a single root have a positive real part, and the corresponding solution is unstable. For zero or imaginary roots, additional analysis would further be explored (Blekhman, 1998).

\subsection{Kinematic equation of the vibrating system}

The model of the vibration system is shown in Fig. 1. The system is mainly composed of three induction motors, coupling unit, crossbeam, screen frame, motor seat. And two motors rotate in the same direction connected with the coupling unit, which consists of a connecting rod, chutes, coupling springs and slide blocks. The chute, linked to the end of the connecting rod by welding, should be mutually parallel. The slide blocks and the coupling springs are installed in the chutes. Besides, the stiffness of the connecting rod is bigger than the coupling springs, and the connecting rod has smaller density. The cross-section area of the connecting rod changes with stiffness of the simplified spring.

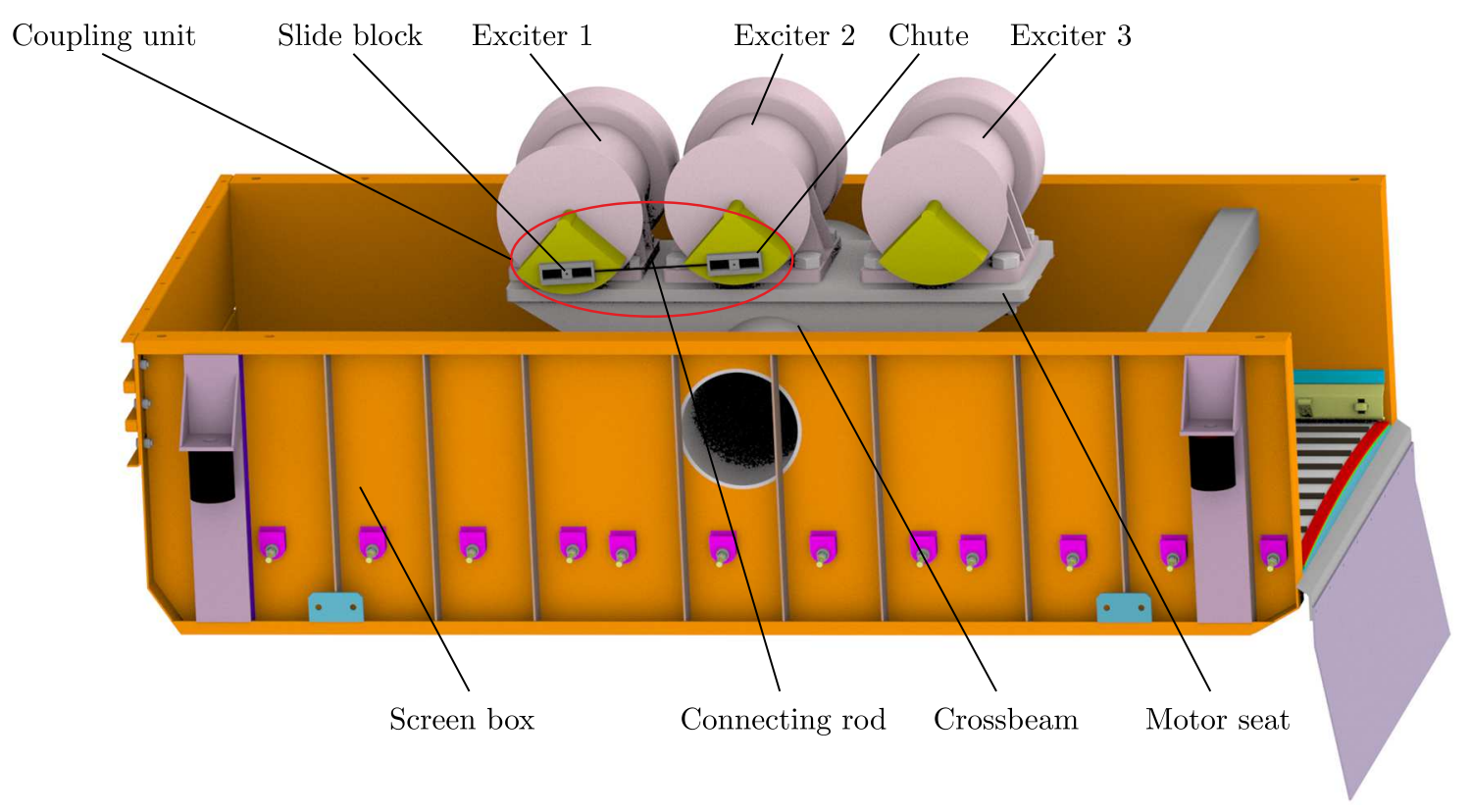

Fig. 1. The model of an elastically coupled tri-rotor system

Figure 2 describes the dynamical model of the considered model. The exciters $m_{i}$ $(i=1,2,3)$ are installed in the screen frame. The rigid vibro-body $m_{0}$ is supported on an elastic foundation by some stronger stiffness springs $k_{x}, k_{y}, k_{\psi}$ in $x-, y$-, $\psi$-directions. The foundation is characterized by damping constants $C_{x}, C_{y}, C_{\psi}$. The elastic coupling unit is simplified as a linear spring $k$, and the distance between the point of connecting of the springs and the motors axles is assumed to be $a$. As illustrated in Fig. 2b, the mass centers of the rigid vibro-body 
is the point $o$. Three reference coordinate system of the vibration system is designed as follows: the non-rotating moving frame $o^{\prime} x^{\prime} y^{\prime}$ is always parallel to the fixed coordinate frame $o x y$ in the $x$ - and $y$-directions, and the moving frame $o^{\prime} x^{\prime \prime} y^{\prime \prime}$ swings around the point $o^{\prime}$. The exciters also rotate around their own spin axes, which are denoted by $\varphi_{i}(i=1,2,3) . M$ is mass of the system, and the installation angle of the motor is expressed by $\beta_{i}(i=1,2,3)$. The responses $x, y$ and the angular rotation $\psi$ are considered as independent coordinates.

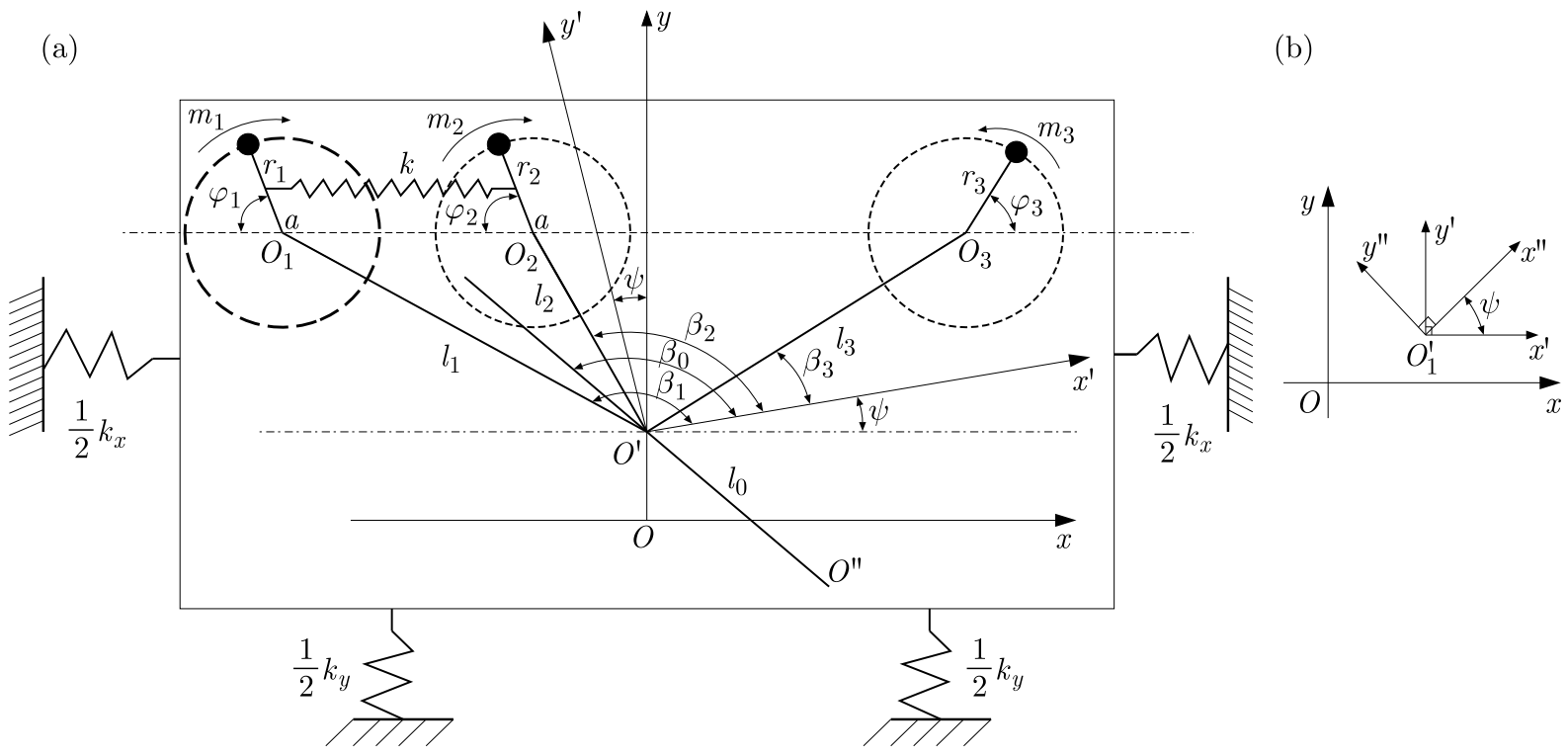

Fig. 2. Simplified model: (a) dynamic model of three rotors coupled with a weak spring, (b) the reference frame system

The expressions for the kinetic energy of the system can be written as follows

$$
\begin{aligned}
T= & \frac{1}{2} m_{0}\left\{\left[\dot{x}-\ell_{0} \dot{\psi} \sin \left(\beta_{0}+\psi+\pi\right)\right]^{2}+\left[\dot{y}+\ell_{0} \dot{\psi} \cos \left(\beta_{0}+\psi+\pi\right)\right]^{2}\right\}+\frac{1}{2} \sum_{i=1}^{3} J_{i} \dot{\varphi}_{i}^{2} \\
& +\frac{1}{2} \sum_{i=1}^{2} m_{i}\left\{\left[\dot{x}-\ell_{i} \dot{\psi} \sin \left(\beta_{i}+\psi\right)+r_{i} \dot{\varphi}_{i} \sin \varphi_{i}\right]^{2}\right. \\
& \left.+\left[\dot{y}+\ell_{i} \dot{\psi} \cos \left(\beta_{i}+\psi\right)+r_{i} \dot{\varphi}_{i} \cos \varphi_{i}\right]^{2}\right\}+\frac{1}{2} m_{3}\left\{\left[\dot{x}-\ell_{3} \dot{\psi} \sin \left(\beta_{3}+\psi\right)-r_{3} \dot{\varphi}_{3} \sin \varphi_{3}\right]^{2}\right. \\
& \left.+\left[\dot{y}+\ell_{3} \dot{\psi} \cos \left(\beta_{3}+\psi\right)+r_{3} \dot{\varphi}_{3} \cos \varphi_{3}\right]^{2}\right\}+\frac{1}{2} J_{0} \dot{\psi}^{2}
\end{aligned}
$$

Moreover, considering that the distance of the co-rotating induction motors is $r$, and assuming that the ratio $(a / r \ll 1)$ is infinitesimally small, the elongation of the coupled spring can be obtained

$$
\Delta \ell=\ell-\ell_{0} \approx a\left(\cos \varphi_{1}-\cos \varphi_{2}\right)
$$

And the potential energy of the system can be written as

$$
V=\frac{1}{2} k_{x} x^{2}+\frac{1}{2} k_{y} y^{2}+\frac{1}{2} k_{\psi} \psi^{2}+\frac{1}{2} \Delta \ell^{2}
$$

In addition, the viscous dissipation function of the vibration system can be expressed as

$$
D=\frac{1}{2} C_{x} \dot{x}^{2}+\frac{1}{2} C_{y} \dot{y}^{2}+\frac{1}{2} C_{\psi} \dot{\psi}^{2}+\frac{1}{2} C_{1} \dot{\varphi}_{1}^{2}+\frac{1}{2} C_{2} \dot{\varphi}_{2}^{2}+\frac{1}{2} C_{3} \dot{\varphi}_{3}^{2}
$$


The dynamics equation of the system can be obtained according to Lagrange's equation

$$
\frac{d}{d t} \frac{\partial(T-V)}{\partial \dot{q}_{i}}-\frac{\partial(T-V)}{\partial q_{i}}+\frac{\partial D}{\partial q_{i}}=Q_{i}
$$

If $q=\left[x, y, \psi, \varphi_{1}, \varphi_{2}, \varphi_{3}\right]^{\mathrm{T}}$ is chosen as the generalized coordinates, the generalized forces are $Q_{x}=Q_{y}=Q_{\psi}=0, Q_{\varphi i}=T_{m i}-T_{f i}$. It can be seen that $m_{i} \ll m_{0}$ and $\psi \ll 1$ in the system, and the inertia coupling from asymmetry of the system can be neglected. Considering $M=\sum_{i=1}^{3} m_{i}+m_{0}, m_{1}=m_{2}, r_{1}=r_{2}$, the kinetic equation of the vibration system is derived as

$$
\begin{aligned}
& M \ddot{x}+C_{x} \dot{x}+k_{x} x=m_{3} r_{3}\left(\ddot{\varphi}_{3} \sin \varphi_{3}+\dot{\varphi}_{3}^{2} \cos \varphi_{3}\right)-\sum_{i=1}^{2} m_{i} r_{i}\left(\ddot{\varphi}_{i} \sin \varphi_{i}+\dot{\varphi}_{i}^{2} \cos \varphi_{i}\right) \\
& M \ddot{y}+C_{y} \dot{y}+k_{y} y=m_{3} r_{3}\left(\dot{\varphi}_{3}^{2} \sin \varphi_{3}-\ddot{\varphi}_{3} \cos \varphi_{3}\right)+\sum_{i=1}^{2} m_{i} r_{i}\left(\dot{\varphi}_{i}^{2} \sin \varphi_{i}-\ddot{\varphi}_{i} \cos \varphi_{i}\right) \\
& J \ddot{\psi}+C_{\psi} \dot{\psi}+k_{\psi} \psi=\sum_{i=1}^{2} m_{i} \ell_{i} r_{i}\left[\dot{\varphi}_{i}^{2} \sin \left(\varphi_{i}+\beta_{i}+\psi\right)-\ddot{\varphi}_{i} \cos \left(\varphi_{i}+\beta_{i}+\psi\right)\right] \\
& \quad+m_{3} r_{3} \ell_{3}\left[\dot{\varphi}_{3}^{2} \sin \left(\varphi_{3}-\beta_{3}-\psi\right)-\ddot{\varphi}_{3} \cos \left(\varphi_{3}-\beta_{3}-\psi\right)\right] \\
& \quad+C_{3}\left(\dot{\varphi}_{3}-\dot{\psi}\right)-C_{1}\left(\dot{\varphi}_{1}+\dot{\psi}\right)-C_{2}\left(\dot{\varphi}_{2}+\dot{\psi}\right) \\
& J_{o 1} \ddot{\varphi}_{1}=T_{m 1}-T_{f 1}-C_{1}\left(\dot{\varphi}_{1}+\dot{\psi}\right)-m_{1} r_{1}\left[\ddot{x} \sin \varphi_{1}+\ddot{y} \cos \varphi_{1}\right] \\
& \quad+m_{1} r_{1} \ell_{1}\left[\dot{\psi}^{2} \sin \left(\varphi_{1}+\beta_{1}+\psi\right)-\ddot{\psi} \cos \left(\varphi_{1}+\beta_{1}+\psi\right)\right]-k a^{2}\left(\cos \varphi_{2}-\sin \varphi_{1}\right) \sin \varphi_{1} \\
& J_{o 2} \ddot{\varphi}_{2}=T_{m 2}-T_{f 2}-C_{2}\left(\dot{\varphi}_{2}+\dot{\psi}\right)-m_{2} r_{2}\left[\ddot{x} \sin \varphi_{2}+\ddot{y} \cos \varphi_{2}\right] \\
& \quad+m_{2} r_{2} \ell_{2}\left[\dot{\psi}^{2} \sin \left(\varphi_{2}+\beta_{2}+\psi\right)-\ddot{\psi} \cos \left(\varphi_{2}+\beta_{2}+\psi\right)\right]-k a^{2}\left(\cos \varphi_{1}-\sin \varphi_{2}\right) \sin \varphi_{2} \\
& J_{03} \ddot{\varphi}_{3}=T_{m 3}-T_{f 3}-C_{3}\left(\dot{\varphi}_{3}-\dot{\psi}\right)+m_{3} r_{3}\left[\ddot{x} \sin \varphi_{3}-\ddot{y} \cos \varphi_{3}\right] \\
& \quad+m_{3} r_{3} \ell_{3}\left[-\dot{\psi}^{2} \sin \left(\varphi_{3}-\beta_{3}-\psi\right)-\ddot{\psi} \cos \left(\varphi_{3}-\beta_{3}-\psi\right)\right]
\end{aligned}
$$

\section{Criterion of synchronization and stability of synchronous states}

\subsection{Method description}

According to the Poincaré method (i.e., based on fundamental Eq. (2.1)), introducing the small parameter $\mu$ into Eq. (2.12), the influence of the small parameter can be ignored, then a new form of Eq. (2.12) is given

$$
\begin{aligned}
& M \ddot{x}+k_{x} x=m_{3} r_{3}\left(\ddot{\varphi}_{3} \sin \varphi_{3}+\dot{\varphi}_{3}^{2} \cos \varphi_{3}\right)-\sum_{i=1}^{2} m_{i} r_{i}\left(\ddot{\varphi}_{i} \sin \varphi_{i}+\varphi_{i}^{2} \cos \varphi_{i}\right) \\
& M \ddot{y}+k_{y} y=m_{3} r_{3}\left(\dot{\varphi}_{3}^{2} \sin \varphi_{3}-\ddot{\varphi}_{3} \cos \varphi_{3}\right)+\sum_{i=1}^{2} m_{i} r_{i}\left(\dot{\varphi}_{i}^{2} \sin \varphi_{i}-\ddot{\varphi}_{i} \cos \varphi_{i}\right) \\
& J \ddot{\psi}+k_{\psi} \psi=\sum_{i=1}^{2} m_{i} \ell_{i} r_{i}\left[\dot{\varphi}_{i}^{2} \sin \left(\varphi_{i}+\beta_{i}+\psi\right)-\ddot{\varphi}_{i} \cos \left(\varphi_{i}+\beta_{i}+\psi\right)\right] \\
& +m_{3} r_{3} \ell_{3}\left[\dot{\varphi}_{3}^{2} \sin \left(\varphi_{3}-\beta_{3}-\psi\right)-\ddot{\varphi}_{3} \cos \left(\varphi_{3}-\beta_{3}-\psi\right)\right]+C_{3} \dot{\varphi}_{3}-C_{1} \dot{\varphi}_{1}-C_{2} \dot{\varphi}_{2} \\
& J_{o 1} \ddot{\phi}_{1}=\mu \phi_{1} \quad J_{o 2} \ddot{\phi}_{2}=\mu \phi_{2} \quad J_{03} \ddot{\phi}_{3}=\mu \phi_{3}
\end{aligned}
$$


where

$$
\begin{aligned}
& \mu \phi_{1}=T_{m 1}-T_{f 1}-m_{1} r_{1}\left[\ddot{x} \sin \varphi_{1}+\ddot{y} \cos \varphi_{1}\right] \\
& \quad+m_{1} r_{1} \ell_{1}\left[\dot{\psi}^{2} \sin \left(\varphi_{1}+\beta_{1}+\psi\right)-\ddot{\psi} \cos \left(\varphi_{1}+\beta_{1}+\psi\right)\right]-k a^{2}\left(\cos \varphi_{2}-\sin \varphi_{1}\right) \sin \varphi_{1} \\
& \mu \phi_{2}=T_{m 2}-T_{f 2}-m_{2} r_{2}\left[\ddot{x} \sin \varphi_{2}+\ddot{y} \cos \varphi_{2}\right] \\
& \quad+m_{2} r_{2} \ell_{2}\left[\dot{\psi}^{2} \sin \left(\varphi_{2}+\beta_{2}+\psi\right)-\ddot{\psi} \cos \left(\varphi_{2}+\beta_{2}+\psi\right)\right]-k a^{2}\left(\cos \varphi_{1}-\sin \varphi_{2}\right) \sin \varphi_{2} \\
& \mu \phi_{3}=T_{m 3}-T_{f 3}+m_{3} r_{3}\left[\ddot{x} \sin \varphi_{3}-\ddot{y} \cos \varphi_{3}\right] \\
& \quad+m_{3} r_{3} \ell_{3}\left[-\dot{\psi}^{2} \sin \left(\varphi_{3}-\beta_{3}-\psi\right)-\ddot{\psi} \cos \left(\varphi_{3}-\beta_{3}-\psi\right)\right]
\end{aligned}
$$

Solving Eq. (3.1), the steady responses in the $x$-, $y$ - and $\psi$-directions are obtained

$$
\begin{aligned}
& x=a_{3} \cos \varphi_{3}-a_{1} \cos \varphi_{1}-a_{2} \cos \varphi_{2} \\
& y=b_{1} \sin \varphi_{1}+b_{2} \sin \varphi_{2}+b_{3} \sin \varphi_{3} \\
& \psi=c_{1} \sin \left(\varphi_{1}+\beta_{1}\right)+c_{2} \sin \left(\varphi_{2}+\beta_{2}\right)+c_{3} \sin \left(\varphi_{3}+\beta_{3}\right)
\end{aligned}
$$

where

$$
\alpha_{i}=\frac{m_{i} r_{i} \dot{\varphi}_{i}^{2}}{k_{x}-M \dot{\varphi}_{i}^{2}} \quad b_{i}=\frac{m_{i} r_{i} \dot{\varphi}_{i}^{2}}{k_{y}-M \dot{\varphi}_{i}^{2}} \quad c_{i}=\frac{m_{i} r_{i} \dot{\varphi}_{i}^{2} \ell_{i}}{k_{\psi}-J \dot{\varphi}_{i}^{2}} \quad i=1,2,3
$$

Here, introducing the following dimensionless parameters, the standard mass $m$ is defined, and the natural frequencies are denoted by $\omega_{x}, \omega_{y}, \omega_{\varphi}$ in the $x-, y$-, $\psi$-direction, respectively.

$$
\begin{array}{lllll}
\eta_{1}=\frac{m_{1}}{m} & \eta_{2}=\frac{m_{2}}{m} & \eta_{3}=\frac{m_{3}}{m} & r_{m}=\frac{m}{M} & r_{e}=\frac{m}{J} \\
\omega_{x}=\sqrt{\frac{k_{x}}{M}} & \omega_{y}=\sqrt{\frac{k_{y}}{M}} & \omega_{\psi}=\sqrt{\frac{k_{\psi}}{J}} & \sigma=\frac{r_{e}}{r_{m}} & \rho=\frac{r_{3}}{r_{1}} \\
\lambda_{1}=\frac{\omega_{m}^{2}}{\omega_{m}^{2}-\omega_{x}^{2}} & \lambda_{2}=\frac{\omega_{m}^{2}}{\omega_{m}^{2}-\omega_{y}^{2}} & \lambda_{3}=\frac{\omega_{m}^{2}}{\omega_{m}^{2}-\omega_{\psi}^{2}} & &
\end{array}
$$

Consequently, basic Eq. (3.3) will be written as

$$
\begin{aligned}
& x=r_{m} \lambda_{1}\left(\eta_{1} r_{1} \cos \varphi_{1}+\eta_{2} r_{2} \cos \varphi_{2}-\eta_{3} r_{3} \cos \varphi_{3}\right) \\
& y=-r_{m} \lambda_{2}\left(\eta_{1} r_{1} \sin \varphi_{1}+\eta_{2} r_{2} \sin \varphi_{2}+\eta_{3} r_{3} \sin \varphi_{3}\right) \\
& \psi=-r_{e} \lambda_{3}\left[\eta_{1} r_{1} \sin \left(\varphi_{1}+\beta_{1}\right)+\eta_{2} r_{2} \sin \left(\varphi_{2}+\beta_{2}\right)+\eta_{3} r_{3} \sin \left(\varphi_{3}-\beta_{3}\right)\right]
\end{aligned}
$$

\subsection{Synchronization criterion}

Theoretical derivation of the synchronization condition is discussed in this Section. Assume that $\alpha_{i}, \varphi_{i}$ are the initial phase and phase angle of the unbalanced rotor $i$, respectively. The solution mentioned above is corresponding with Eq. (2.3)

$$
\varphi_{1}=\omega t+\alpha_{1} \quad \varphi_{2}=\omega t+\alpha_{2} \quad \varphi_{3}=\omega t+\alpha_{3}
$$


According to Eq. (2.4), substituting Eq. (3.7) into Eq.(3.2), $P_{i}$ can be calculated

$$
\begin{aligned}
P_{1} & =\left\langle\mu \phi_{1}\right\rangle=T_{m 1}-T_{f 1}-\frac{1}{2} m_{1} r_{m} r_{1} \omega^{2}\left[\eta_{2} r_{2}\left(\lambda_{1}+\lambda_{2}\right) \sin \left(\alpha_{2}-\alpha_{1}\right)\right. \\
& \left.+\eta_{3} r_{3}\left(\lambda_{2}-\lambda_{1}\right) \sin \left(\alpha_{3}-\alpha_{1}\right)\right]-\frac{1}{2} m_{1} r_{1} \ell_{1} r_{e} \lambda_{3} \omega^{2}\left[\eta_{2} r_{2} \sin \left(\alpha_{2}-\alpha_{1}+\beta_{2}-\beta_{1}\right)\right. \\
& \left.+\eta_{3} r_{3} \sin \left(\alpha_{3}-\alpha_{1}-\beta_{3}-\beta_{1}\right)\right]+\frac{1}{2} k a^{2}\left[\sin \left(\alpha_{2}-\alpha_{1}\right)+1\right]=0 \\
P_{2} & =\left\langle\mu \phi_{2}\right\rangle=T_{m 2}-T_{f 2}-\frac{1}{2} m_{2} r_{m} r_{2} \omega^{2}\left[-\eta_{1} r_{1}\left(\lambda_{1}+\lambda_{2}\right) \sin \left(\alpha_{2}-\alpha_{1}\right)\right. \\
& \left.+\eta_{3} r_{3}\left(\lambda_{1}-\lambda_{2}\right) \sin \left(\alpha_{2}-\alpha_{3}\right)\right]-\frac{1}{2} m_{2} r_{2} \ell_{2} r_{e} \lambda_{3} \omega^{2}\left[\eta_{1} r_{1} \sin \left(\alpha_{1}-\alpha_{2}+\beta_{1}-\beta_{2}\right)\right. \\
& \left.+\eta_{3} r_{3} \sin \left(\alpha_{3}-\alpha_{2}-\beta_{3}-\beta_{2}\right)\right]+\frac{1}{2} k a^{2}\left[-\sin \left(\alpha_{2}-\alpha_{1}\right)+1\right]=0 \\
P_{3} & =\left\langle\mu \phi_{3}\right\rangle=T_{m 3}-T_{f 3}+\frac{1}{2} m_{3} r_{m} r_{3} \omega^{2}\left[\eta_{1} r_{1}\left(\lambda_{1}-\lambda_{2}\right) \sin \left(\alpha_{1}-\alpha_{3}\right)\right. \\
& \left.+\eta_{2} r_{2}\left(\lambda_{1}-\lambda_{2}\right) \sin \left(\alpha_{2}-\alpha_{3}\right)\right] \\
& -\frac{1}{2} m_{3} r_{3} \ell_{3} r_{e} \lambda_{3} \omega^{2}\left[\eta_{1} r_{1} \sin \left(\alpha_{1}-\alpha_{3}+\beta_{1}+\beta_{3}\right)+\eta_{2} r_{2} \sin \left(\alpha_{2}-\alpha_{3}+\beta_{2}+\beta_{3}\right)\right]=0
\end{aligned}
$$

When the angular velocity of the tri-rotors is near to the synchronous velocity $\omega_{m}$, the excessive torque $Z_{s}(\omega)$ of the rotors is equal to zero in the synchronization state

$$
Z_{i}(\omega)=T_{m i}-T_{f i}=0 \quad i=1,2,3
$$

The balance equation of synchronization of the vibrating system can be expressed as

$$
\begin{aligned}
& \mu_{1}\left[\sin \left(\alpha_{3}-\alpha_{1}\right)+\sin \left(\alpha_{3}-\alpha_{2}\right)\right]+\mu_{2} \sin \left(\alpha_{2}-\alpha_{1}+\beta_{2}-\beta_{1}\right) \\
& \quad+\mu_{3} \sin \left(\alpha_{3}-\alpha_{1}-\beta_{3}-\beta_{1}\right)+\mu_{4} \sin \left(\alpha_{3}-\alpha_{2}-\beta_{3}-\beta_{2}\right)-\mu_{7}=0 \\
& \mu_{5}\left[\sin \left(\alpha_{1}-\alpha_{3}\right)+\sin \left(\alpha_{2}-\alpha_{3}\right)\right]+\mu_{6}\left[\sin \left(\alpha_{3}-\alpha_{1}-\beta_{1}-\beta_{3}\right)\right. \\
& \left.\quad+\sin \left(\alpha_{3}-\alpha_{2}-\beta_{2}-\beta_{3}\right)\right]=0
\end{aligned}
$$

where

$$
\begin{array}{lll}
\mu_{1}=\eta_{1} \eta_{3} \rho\left(\lambda_{2}-\lambda_{1}\right) & \mu_{2}=\eta_{1} \eta_{2} \sigma \lambda_{3}\left(\ell_{1}-\ell_{2}\right) & \mu_{3}=\eta_{1} \eta_{3} \ell_{1} \sigma \rho \lambda_{3} \\
\mu_{4}=\eta_{2} \eta_{3} \ell_{2} \sigma \rho \lambda_{3} & \mu_{5}=\lambda_{1}-\lambda_{2} & \mu_{6}=\ell_{3} \sigma \lambda_{3} \\
\mu_{7}=\frac{2 k a^{2}}{m_{0} r_{1}^{2} r_{m} \omega^{2}} & &
\end{array}
$$

\subsection{Stability criterion of synchronization states}

Introduce now new parameters $A, B, C, D$, i.e:

$$
A=\frac{\partial\left(P_{1}-P_{3}\right)}{\partial \alpha_{1}} \quad B=\frac{\partial\left(P_{2}-P_{3}\right)}{\partial \alpha_{2}} \quad C=\frac{\partial\left(P_{2}-P_{3}\right)}{\partial \alpha_{1}} \quad D=\frac{\partial\left(P_{2}-P_{3}\right)}{\partial \alpha_{2}}
$$

According to Eq.(2.6), the stability criterion of synchronization of the system can be expressed as

$$
A+B<0
$$


Inserting Eq. (3.8) and Eq. (3.12) into Eq. (3.13), the stability criterion of synchronization states can be simplified as

$$
\begin{aligned}
& 2 \mu_{8} \cos \left(\alpha_{2}-\alpha_{1}\right)+2 \mu_{1}\left[\cos \left(\alpha_{3}-\varphi_{1}\right)+\cos \left(\alpha_{2}-\alpha_{3}\right)\right]+\mu_{9} \cos \left(\alpha_{2}-\alpha_{1}+\beta_{2}-\beta_{1}\right) \\
& \quad+\mu_{10} \cos \left(\alpha_{1}-\alpha_{3}+\beta_{1}+\beta_{3}\right)+\mu_{11} \cos \left(\alpha_{2}-\alpha_{3}+\beta_{2}+\beta_{3}\right)-\mu_{7} \cos \left(\alpha_{2}-\alpha_{1}\right)<0
\end{aligned}
$$

where

$$
\begin{array}{ll}
\mu_{8}=\eta_{1} \eta_{2}\left(\lambda_{1}+\lambda_{2}\right) & \mu_{9}=\eta_{1} \eta_{2}\left(\ell_{1}+\ell_{2}\right) \sigma \lambda_{3} \\
\mu_{10}=\eta_{1} \eta_{3} \rho \sigma \lambda_{3}\left(\ell_{1}+\ell_{3}\right) & \mu_{11}=\eta_{1} \eta_{2} \rho \sigma \lambda_{3}\left(\ell_{2}+\ell_{3}\right)
\end{array}
$$

Therefore, only the system parameters satisfy balance equations (3.10) and the stability criterion of synchronization (3.14) can be implemented in the considered case.

\section{Numerical verification}

In the above Sections, the differential equations, balanced equations and the stability criterion of synchronization have been derived. The theoretical and simulation results are presented in this Section, where the correctness of the theory is to be verified.

\subsection{Analysis of numerical results}

Some examples are used to prove the correctness of the results of the above theoretical derivation. Based on Eq. (4.1), the stiffness coefficients $k_{x}, k_{y}$ and $k_{\varphi}$ are separately transformed into frequency ration $\eta_{x}, \eta_{y}, \eta_{\varphi}$. Balance equations (3.10) are nonlinear equations related to the system parameters, including the supporting spring stiffness, stiffness of elastic spring $k$, installation location, etc., which seriously influence the stability of self-synchronization of the system. When the system parameters are simultaneously satisfied, balance equation (3.10), stability criterion (3.14) and the stable phase difference can be estimated by applying a numerical method. In order to simplify calculations, we assume $\eta_{x}=\eta_{y}=\eta_{\varphi}$, i.e.,

$$
\eta_{x}=\frac{\omega}{\omega_{x}} \quad \eta_{y}=\frac{\omega}{\omega_{y}} \quad \eta_{\varphi}=\frac{\omega}{\omega_{\varphi}}
$$

Studying synchronization of the vibration system, the parameters are shown in Table 1, and the dimensionless values are shown in Table 2 according to Eq. (3.5).

Table 1. Parameter values of the system

\begin{tabular}{|l|l|}
\hline Unbalanced rotor for $i=1,2,3$ & $m_{i}=3 \mathrm{~kg}, r_{i}=0.02 \mathrm{~m}, \omega_{m}=156 \sim 157 \mathrm{rad} / \mathrm{s}$, \\
& $c_{i}=0.01 \mathrm{~N} \cdot \mathrm{s} / \mathrm{m}$ \\
\hline Vibro-platform & $M=100 \mathrm{~kg} J=10 \mathrm{~kg} \cdot \mathrm{m}^{2}, f_{x}=1000 \mathrm{~N} /(\mathrm{m} / \mathrm{s})$, \\
& $f_{y}=1000 \mathrm{~N} /(\mathrm{m} / \mathrm{s}), f_{z}=1000 \mathrm{~N} /(\mathrm{m} / \mathrm{s})$, \\
& $k_{x}=1 \cdot 10^{4} \sim 3.65 \cdot 10^{5} \mathrm{~N} / \mathrm{m}$, \\
& $k_{y}=1 \cdot 10^{4} \sim 3.65 \cdot 10^{5} \mathrm{~N} / \mathrm{m}$, \\
& $k_{\psi}=1 \cdot 10^{3} \sim 3.65 \cdot 10^{4} \mathrm{~N} / \mathrm{m}$ \\
\hline Other parameters & $l_{1}=0.8 \mathrm{~m}, l_{2}=0.73,0.41 \mathrm{~m}, l_{3}=0.8 \mathrm{~m}$, \\
& $\beta_{1}=2 \pi / 3,5 \pi / 6, \beta_{2}=2 \pi / 5,5 \pi / 12, \beta_{3}=\pi / 3, \pi / 6$ \\
\hline The spring & $k=0 \sim 1.4 \cdot 10^{5} \mathrm{~N} / \mathrm{m}, a=0.01 \mathrm{~m}$ \\
\hline
\end{tabular}

Equations (3.10) and (3.14) describe the approximate analytical solution for the stable phase difference. Based on the parameters in Table 2, we can acquire an approximate value of $\varphi_{1}-\varphi_{2}$ and $\varphi_{1}-\varphi_{3}$ considering different parameters $k, \eta_{x}, \eta_{y}, \eta_{\varphi}$ when three motors are installed in 
Table 2. Parameter values according to dimensionless Eq. (3.5)

$$
\begin{aligned}
& \eta_{1}=1, \eta_{2}=1, \eta_{3}=1, r_{m}=0.02, r_{e}=0.18, \sigma=8.82, \rho=1 \\
& n_{x}=1 \sim 19, n_{y}=1 \sim 19, n_{\varphi}=1 \sim 15.7
\end{aligned}
$$

different positions. The analytical results are shown in Fig. 3 and Fig. 4. They indicate that the parameters $\eta_{x}, \eta_{y}, \eta_{\varphi}$, have little influence on the value of the phase difference when the above-mentioned balance equation and the stability criterion equation are satisfied. But the parameter $k$ directly affects the phase difference. Figure 3 shows numerical results for positional parameters (i.e, $l_{1}=0.8 \mathrm{~m}, l_{2}=0.73 \mathrm{~m}, l_{3}=0.8 \mathrm{~m}, \beta_{1}=2 \pi / 3, \beta_{2}=2 \pi / 5, \beta_{3}=\pi / 3$ ) for different frequency ratios. When $k=0 \mathrm{~N} / \mathrm{m}$ (there is no coupling unit in co-rotating motors), the phase difference $\varphi_{1}-\varphi_{2}$ of the co-rotating motors is stabilized in the vicinity of $3 \mathrm{rad}$, and the phase difference $\varphi_{1}-\varphi_{3}$ of the counter-rotating motors is near $1 \mathrm{rad}$. When $k \geqslant 30000 \mathrm{~N} / \mathrm{m}$, the phase difference of the co-rotating motors is close to $0 \mathrm{rad}$ and the stable difference $\varphi_{1}-\varphi_{3}$ is near $1 \mathrm{rad}$. Meanwhile, the vibration amplitude improves when the stiffness of the coupling spring $k$ exceeds the maximum value $k_{\max }=140000 \mathrm{~N} / \mathrm{m}$ (Fig. 3a), and $k_{\max }=120000 \mathrm{~N} / \mathrm{m}$ (Fig. 3b,c,d), which means that the synchronous motion is unstable. The numerical results for $l_{1}=0.8 \mathrm{~m}, l_{2}=0.41 \mathrm{~m}, l_{3}=0.8 \mathrm{~m}, \beta_{1}=5 \pi / 6, \beta_{2}=5 \pi / 12, \beta_{3}=\pi / 6$ are displayed in Fig. 4. Similar conclusions are also obtained.
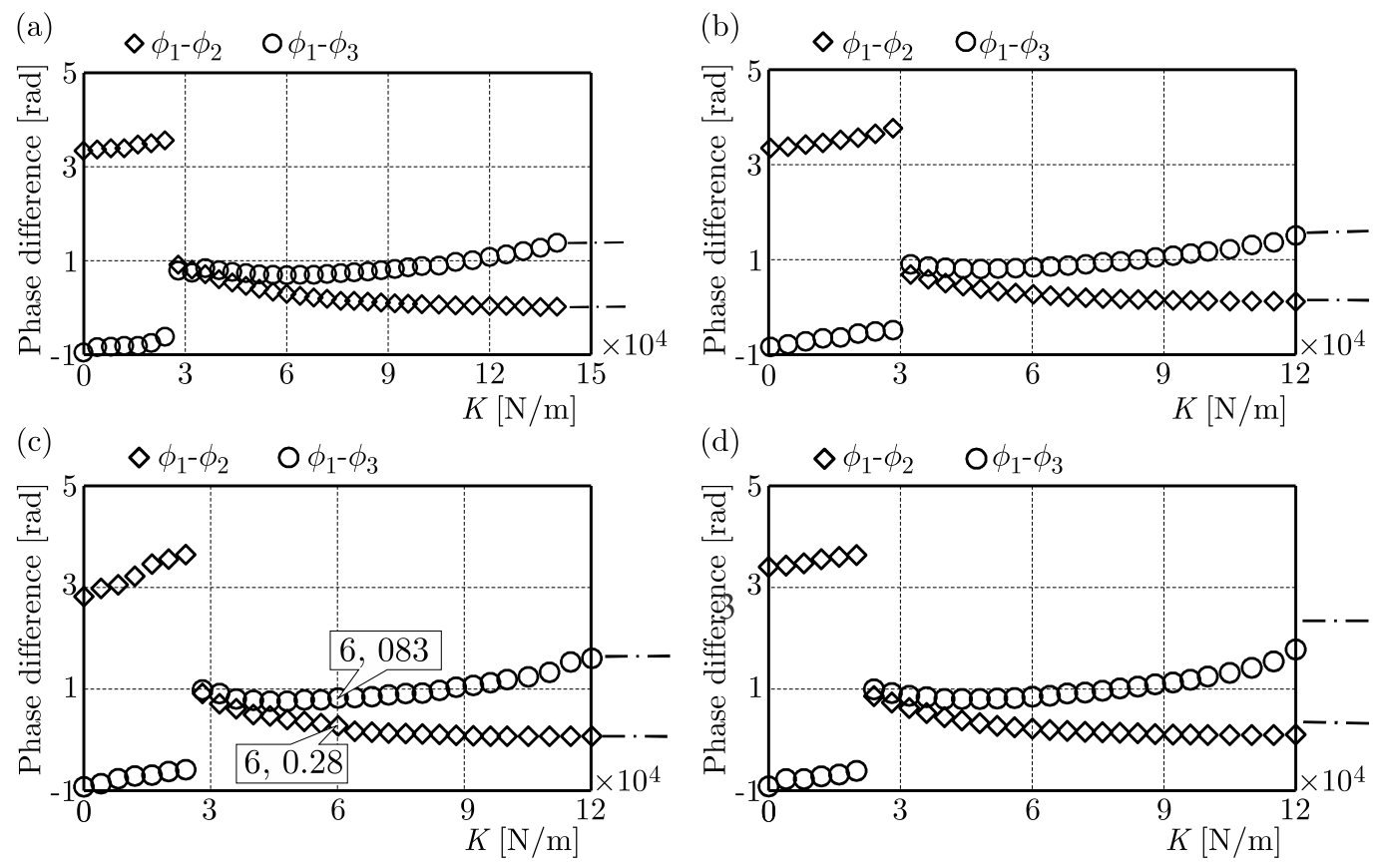

Fig. 3. Stable phase difference with theoretical analysis for $l_{1}=0.8 \mathrm{~m}, l_{2}=0.73 \mathrm{~m}, l_{3}=0.8 \mathrm{~m}$, $\beta_{1}=2 \pi / 3, \beta_{2}=2 \pi / 5, \beta_{3}=\pi / 3 ;$ (a) $\eta_{x}=\eta_{y}=\eta_{\varphi}=1.76$, (b) $\eta_{x}=\eta_{y}=\eta_{\varphi}=3.51$,

(c) $\eta_{x}=\eta_{y}=\eta_{\varphi}=5.23$, (d) $\eta_{x}=\eta_{y}=\eta_{\varphi}=7.85 ;-\cdot-$ shows there is no stable phase difference

The above analysis implies that these parameters play an important role in the synchronous state, which mainly include the stiffness coefficient $k$, frequency ratios and installation location of three induction motors. Besides, the coupled spring connecting the co-rotation rotors is also compliant with the condition and stability of synchronization. By selecting a large value of $k$, the vibration amplitude and the screening efficiency of the system can be improved.

\subsection{Buckling analysis of the connecting rod}

The two chutes are connected by the connecting rod. During the process of self-synchronization, the elasticity coupling between the two induction motors can be achieved 

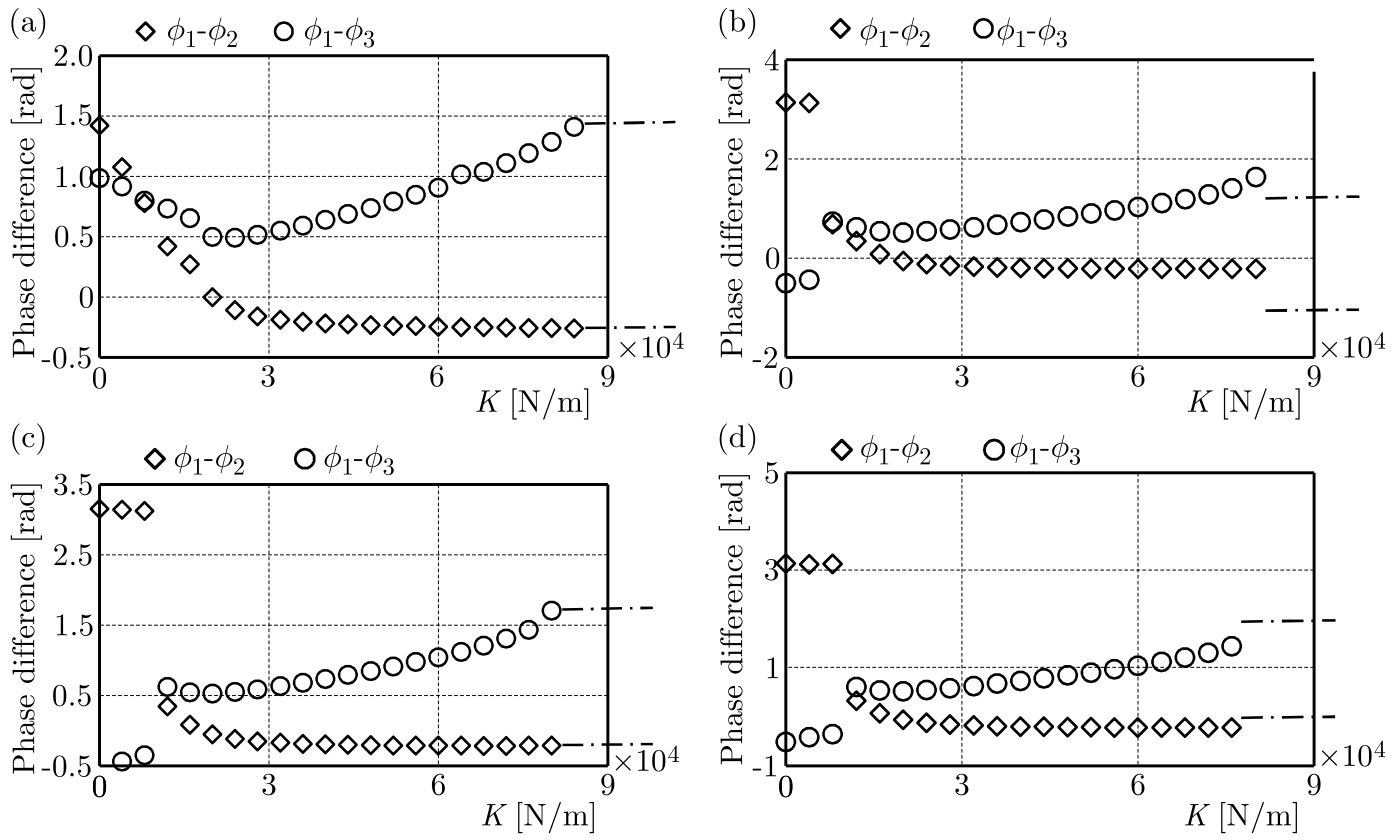

Fig. 4. Stable phase difference with theoretical analysis for $l_{1}=0.8 \mathrm{~m}, l_{2}=0.41 \mathrm{~m}, l_{3}=0.8 \mathrm{~m}$, $\beta_{1}=5 \pi / 6, \beta_{2}=5 \pi / 12, \beta_{3}=\pi / 6 ;$ (a) $\eta_{x}=\eta_{y}=\eta_{\varphi}=2.22$, (b) $\eta_{x}=\eta_{y}=\eta_{\varphi}=4.96$

(c) $\eta_{x}=\eta_{\varphi}=5.55$, (d) $\eta_{x}=\eta_{y}=\eta_{\varphi}=7.02,-\cdot-$ shows there is no stable phase difference

by the springs in the chutes. Owing to the connecting rod with smaller density and the strong stiffness, the centrifugal inertia force of the rod is small, in which case a deflection of the elastic rod can be ignored. For example, the location parameters of vibration motors are identical with the parameters in Fig. 3. According to theoretical analysis (Fig. 3), the stiffness of the simplified spring has the maximum value $k_{\max }=120000 \mathrm{~N} / \mathrm{m}$. Assume $k=80000 \mathrm{~N} / \mathrm{m}$ in this case.

The phase difference of co-rotating motors is expressed as $\alpha$, which satisfies

$$
\alpha=\left|\varphi_{1}-\varphi_{2}\right|
$$

The range of the phase difference $\alpha$ is obtained as $0 \leqslant \alpha \leqslant \pi$. When $\alpha=0^{\circ}$, the deformation of the simplified spring is equal to 0 ; when $\alpha=180^{\circ}$, the deformation of the simplified spring has the maximum value, $x_{\max }=2 a=0.02 \mathrm{~m}$ (the simplified spring is in stretched state or under compression), $0 \leqslant x \leqslant x_{\max }$.

The force in the connecting rod satisfies

$$
F=k x \leqslant F_{\text {max }}=k x_{\max }=80000 \frac{\mathrm{N}}{\mathrm{m}} \cdot 0.02 \mathrm{~m}=1600 \mathrm{~N}
$$

Assuming that the material of the rod is 2024(LY12), then the yield strength and the density are $[\sigma]=325 \mathrm{MPa}, \rho=2770 \mathrm{~kg} \cdot \mathrm{m}^{-3}, E=72 \mathrm{GPa}=7.2 \cdot 10^{4} \mathrm{~N} / \mathrm{mm}^{2}$. Then the cross-section area $A$ of the connecting rod can be determined

$$
A \geqslant \frac{F_{\max }}{[\sigma]}=4.92 \mathrm{~mm}^{2}
$$

The model of the connecting rod in buckling analysis is shown in Fig. 5. The applied load is expressed as $F(\mu=1)$. The inertia moment of circular section can be expressed as

$$
I=\frac{1}{32} \pi d^{4}
$$




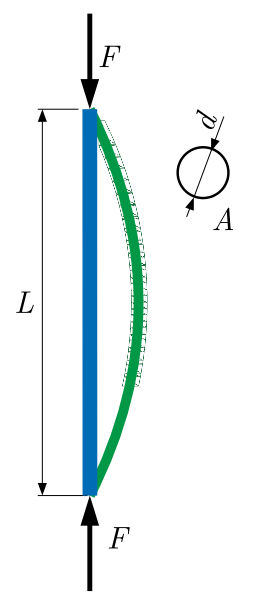

Fig. 5. The model of the connecting rod in buckling analysis

The cross-sectional area $A$ of the connecting rod

$$
A=\frac{\pi d^{2}}{4}
$$

The critical load of the rod can be computed by Euler's formula

$$
F_{c r}=\frac{\pi^{2} E I}{(\mu L)^{2}}
$$

If the buckling of the rod is not achieved, the statical criterion for elastic stability satisfies

$$
F<F_{c r}
$$

Based on the critical condition $F=F_{\max }$, from equation (4.5)-(4.8), $A$ can be calculated

$$
A>\sqrt{\frac{2 F_{\max }(\mu L)^{2}}{E \pi}}=35.7 \mathrm{~mm}^{2}
$$

Therefore, the cross-section area the connecting rod $A$ can be obtained

$$
A>35.7 \mathrm{~mm}^{2}
$$

If $A=36 \mathrm{~mm}^{2}$, so the mass can be calculated

$$
m=\rho L S=2770 \frac{\mathrm{kg}}{\mathrm{m}^{2}} \cdot 0.3 \mathrm{~m} \cdot 36 \mathrm{~mm}^{2}=0.03 \mathrm{~kg} \ll M=100 \mathrm{~kg}
$$

Therefore, the mass is too small to be neglected.

According to the national design standard, the size of the coupling springs can be finally established.

4.3. Simulation results for $n_{x}=n_{y}=n_{\varphi}=5.23, k=60000 \mathrm{~N} / \mathrm{m}, l_{1}=0.8 \mathrm{~m}, l_{2}=0.73 \mathbf{m}$, $l_{3}=0.8 \mathbf{m}$

Simulation results for the dimensionless parameters in Table 3 are shown in Fig. 6. Here, $k_{x}=k_{y}=9.0 \cdot 10^{4} \mathrm{~N} / \mathrm{m}, k_{\psi}=9.0 \cdot 10^{3} \mathrm{~N} / \mathrm{rad}, l_{1}=0.8 \mathrm{~m}, l_{2}=0.73 \mathrm{~m}, l_{3}=0.8 \mathrm{~m}$, and the other parameters are identical with those in Table 1. From Fig. 6a to Fig. 6f, it can be seen that the self-synchronization of the system is implemented. The three induction motors cannot 
Table 3. Dimensionless parameter values

$$
\begin{aligned}
& \eta_{1}=1, \eta_{2}=1, \eta_{3}=1, r_{m}=0.02, r_{e}=0.20, \sigma=8.70, \rho=1, \\
& n_{x}=8.54, n_{y}=8.54, n_{\varphi}=8.54
\end{aligned}
$$

(a)

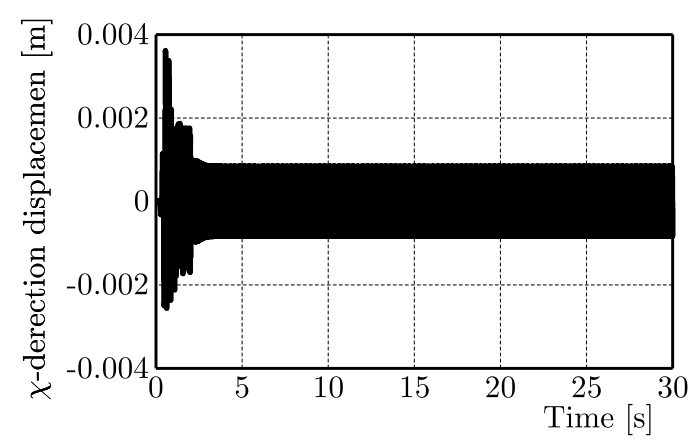

(c)

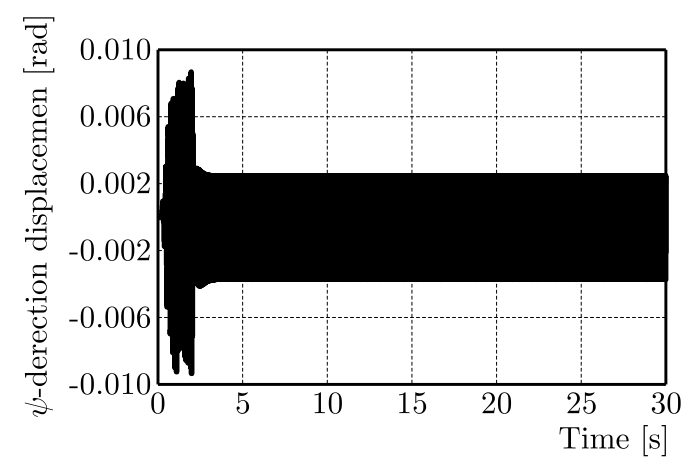

(e)

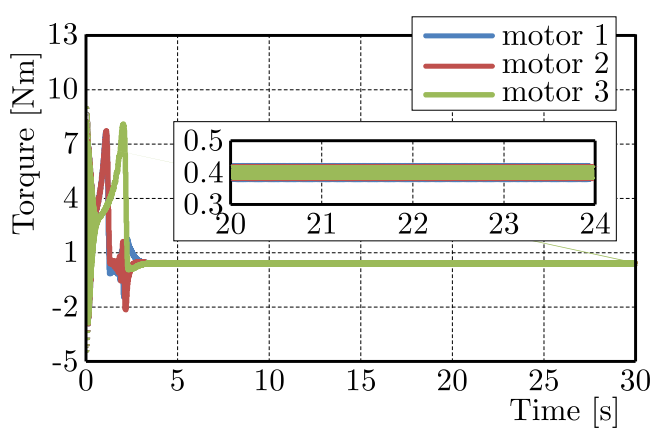

(b)

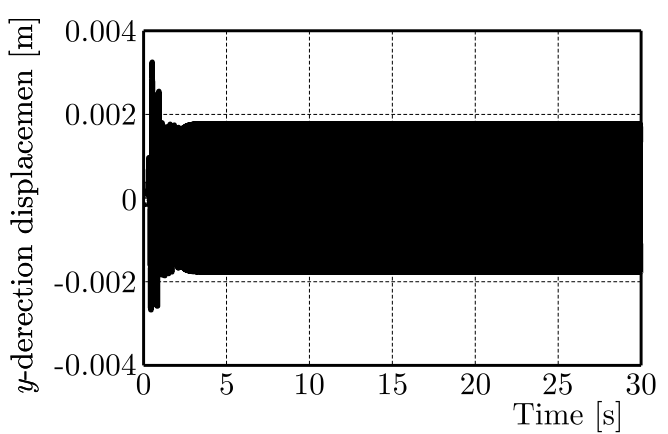

(d)

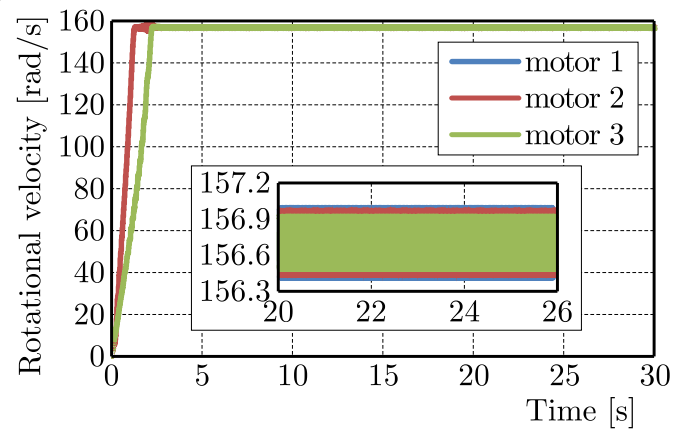

(f)

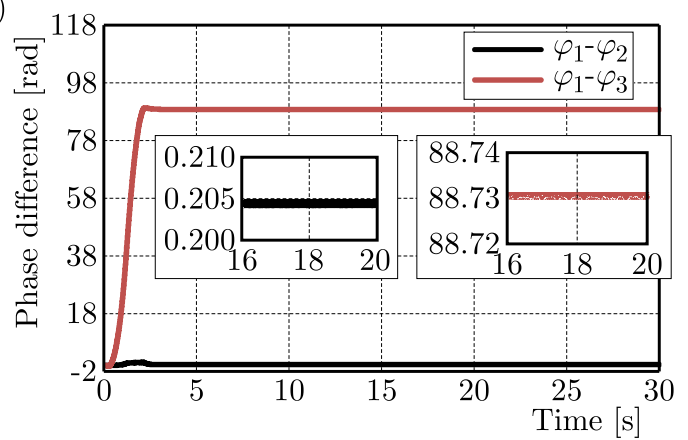

Fig. 6. Results of computer simulations. (a),(b),(c) Displacement responses of the vibrating body in the $x-, y-, \psi$-directions, respectively; (d) rotational velocities of the vibration system; (e) electromagnetic torques of the tri-rotors; (f) phase difference of unbalanced rotors

simultaneously start at the same angular velocity owing to the difference coupling characteristics when three exciters are switched on at the same time. Eventually, the value of angular velocity is identical, see Fig. 6d. The average angular velocity of the three unbalanced rotors is $155.7 \mathrm{rad} / \mathrm{s}$ at about $2 \mathrm{~s}$, which is always defined as the synchronous velocity. In addition, the coupling torques (Fig. 6e), keeping the vibration system working in a steady synchronization state, are approximately $0.4 \mathrm{~N} / \mathrm{m}$. The phase difference $\varphi_{1}-\varphi_{2}$ of the co-rotating motors is near $0.205 \mathrm{rad}$. The phase difference $\varphi_{1}-\varphi_{3}$ of the counter-rotating motors stabilized in the vicinity of $88.73 \mathrm{rad}(88.73 \mathrm{rad}=(28 \pi+0.77) \mathrm{rad}$, Fig. $6 \mathrm{f})$, agrees with the approximate theoretical value $0.83 \mathrm{rad}$. The stable difference $\varphi_{1}-\varphi_{2}$ is equal to $0.28 \mathrm{rad}$, Fig. 3c. It can be seen that the ideal phase synchronization is achieved by two co-rotation rotors coupled with a weak spring, and the excitation forces of the system are improved. The displacement response of the vibrating body is displayed in the $x-, y$ - and $\psi$-directions, respectively, Fig. 6a,b,c. The computer simulation results further proved the validity of theoretical analysis. 


\section{Conclusions}

Based on the theoretical research and numerical analysis, the following conclusions are obtained.

In this paper, a new vibration mechanism, an elastically coupled tri-rotor system, is proposed to implement synchronization. The average method of small parameters is used to study synchronization characteristics of the system. The dynamical equations are converted into dimensionless equations, and the synchronized state have been investigated. When the values of the system parameters satisfy the balance equations and the stability criterion of synchronization, the vibration system can operate in a steady state. The study indicates that many factors, such as the spring stiffness, stiffness of the elastic unit and the installation location, influence stability of the system. Finally, computer simulations have been preformed to verify the correctness of the approximate solution from computations for the vibration system. Besides, it can be found that the spring connecting the co-rotation rotors makes the phase difference stabilized in the vicinity of $0 \mathrm{rad}$, and the vibration amplitude of the system is improved in contrast to the former one. In this case, the screening efficiency of the system can be improved as well. Moreover, when stiffness of the coupling spring exceeds the maximum value, the vibration system locates in an unstable state. In short, a new balanced elliptical vibrating screen is proposed having a bright future in applications.

Acknowledgment

This study has been supported by science and technology support plan of Sichuan province (2016RZ0059) and the National Natural Science Foundation of China (Grant No. 51074132).

\section{References}

1. Balthazar J.M., 2004, Short comments on self-synchronization of two non-ideal sources supported by a flexible portal frame structure, Journal of Vibration and Control, 10, 1739-1748

2. Balthazar J.M., Felix J.L.P., Brasil R.M., 2005, Some comments on the numerical simulation of self-synchronization of four non-ideal exciters, Applied Mathematics and Computation, 164, $615-625$

3. Blekhman I.I., 1988, Synchronization in Science and Technology, ASME Press

4. Blekhman I.I., Fradkov A.L., Nijmeijer H., Pogromsky A.Y., 1997, On self-synchronization and controlled synchronization, Proceedings of European Control Conference

5. Blekhman I.I., Fradkov A.L., Tomchina O.P., Bogdanov D.E., 2002, Self-synchronization and controlled synchronization: general definition and example design, Mathematics and Computers in Simulation, 58, 367-384

6. Fang P., Hou Y., Nan Y., Yu L., 2015, Study of synchronization for a rotor-pendulum system with Poincare method, Journal of Vibroengineering, 17, 2681-2695

7. Fradkov A.L., Andrievsky B., 2007, Synchronization and phase relations in the motion of two-pendulum system, International Journal of Non-Linear Mechanics, 42, 895-901

8. Hou J., 2007, The synchronous theory of three motor self-synchronism exciting elliptical motion shaker, Journl of Southwest Petroleum University, 29, 168-172

9. Huygens C., 1673, Horologium Oscilatorium, Paris, Frence

10. Jovanovic V., Koshkin S., 2012, Synchronization of Huygens' clocks and the Poincaré method, Journal of Sound and Vibration, 331, 2887-2900

11. Koluda P., Perlikowski P., Czolczynski K., Kapitaniak T., 2014, Synchronization configurations of two coupled double pendula, Communications in Nonlinear Science and Numerical Simulation, 19, 977-990 
12. Koluda P., Perlikowski P., Czolczynski K., Kapitaniak T., 2014, Synchronization of two self-excited double pendula, The European Physical Journal Special Topics, 223, 613-629

13. Kumon M., Washizaki R., Sato J., Kohzawa R., Mizumoto I., Iwai Z., 2002, Controlled synchronization of two 1-DOF coupled oscillators, International Journal of Bifurcation and Chaos in Applied Sciences and Engineering, 15, 1

14. Rui D., 2014, Anti-phase synchronization and ergodicity in arrays of oscillators coupled by an elastic force, European Physical Journal Special Topics, 223, 665-676

15. Zhang X.L., Wen B.C., Zhao C.Y., 2012, Synchronization of three homodromy coupled exciters in a non-resonant vibrating system of plane motion, Acta Mechanica Sinica, 28, 1424-1435

16. Zhang X.L., Wen B.C., Zhao C.Y., 2013, Synchronization of three non-identical coupled exciters with the same rotating directions in a far-resonant vibrating system, Journal of Sound and Vibration, 332, 2300-2317

17. Zhao C.Y., Zhang Y.M., Wen B.C., 2010, Synchronisation and general dynamic symmetry of a vibrating system with two exciters rotating in opposite directions, Acta Physica Sinica, 19, 14-20

Manuscript received January 16, 2016; accepted for print July 14, 2016 